\title{
PERFORMANCE EVALUATION OF PROACTIVE, REACTIVE AND HYBRID ROUTING PROTOCOLS WITH MOBILITY MODEL IN
} MANETS

\author{
Sunil Kumar Singh1 ${ }^{1}$, Rajesh Duvvuru ${ }^{2}$, Amit Bhattcharjee ${ }^{3}$ \\ ${ }^{1,2}$ Assistant Professor, Department of Computer Science and Engineering, National Institute of Technology, Jamshedpur, \\ Jharkhand, India, sunilkrsingh.cse@nitjsr.ac.in,rajeshduvvuru.cse $e^{2} @$ nitjsr.ac.in \\ ${ }^{3}$ Assistant Professor, Department of Computer Science and Engineering, RVS College of Engineering \& Technology, \\ Jharkhand, India, amit.23jsr@gmail.com
}

\begin{abstract}
Our work mainly focused on the performance and effects of different mobility models like Random Waypoint, Reference Point Group, and Manhattan mobility models in different aspects to improve and analyze the behavior of Optimized Link-State Routing (OLSR), Temporally-Ordered Routing Algorithm (TORA) and Zone Routing Protocol (ZRP) routing protocols. These three routing protocols can be classified into the following three general categories, based on the timing when the routes are discovered and updatedproactive (OLSR), reactive (TORA) and hybrid (ZRP). In literature various researchers have discussed the performance issues in AODV, DSDV and DSR routing protocols in Random Waypoint mobility model on Mobile Ad hoc Networks (MANETs) is not satisfactory due to link failure and late acknowledgement. To resolve the specified issue, we have come up with other alternatives like Reference Point Group, and Manhattan mobility model and also other routing protocols like OSLR, TORA and ZRP. A simulation was carried out in NS2 and Bonnmotion for above said protocols and mobility models in Constant Bit Rate (CBR) traffic to analyzed using various metrics like packet delivery fraction, end to end delay and normalized routing load. In our simulation it was shown that few mobility model performed better in different routing protocols. In our simulation results, we got a high Normalized Routing Load for Random Waypoint compared to Reference Point Group, and Manhattan mobility model in both DRP and OSLR protocols.
\end{abstract}

Index Terms: MANET, CBR, Routing protocols, Mobility models, NS2 ***

\section{INTRODUCTION}

A mobile ad-hoc network (MANET) is a group of nodes, which are capable to connect without any infrastructure and prior configuration. Communication can take place between various nodes with the help of wireless links; these nodes are also acts as a router. The nodes are Mobiles and free to transmit packets to their neighbors. Direct communication can happen between hosts that are within the communication range of respective hosts; otherwise, communication is accomplished through multi-hop routing. The MANETs are highly useful in areas like conference hall, lecture theatres, battlefields, emergency rescue services, and others places where providing the network services is very tough due to their geographical shape. Their topology/location changes frequently and unpredictably, these networks require efficient routing protocols that can perform better on immediate topological changes. These protocols are classified in three categories pro-active, re-active and Hybrid routing protocols [1]. In real world for analyzing and evaluating the performance of MANETs protocols and dynamic behavior of mobile nodes, various scenarios are used generated by the different mobility models [2]. Mobility models play an important part in the improvement of MANETs. We can improve the performance of routing protocol for efficient packet delivery by using data traffic. In ad-hoc network three types of data traffic are i.e. TCP, CBR and VCR. TCP is trustworthy, connection oriented data traffic but $\mathrm{CBR}$ is connectionless data traffic used in ad-hoc network. Considering various parameters such as mobility, network load, delay and pause time several performance evaluation of MANET routing protocols have been done using CBR traffic patterns.

\section{RELATED WORK}

Analysis expose that TCP performs poorly in MANETs due to misinterpretation of packet losses, link failure, and late acknowledgement. A. Pal. et. al. [3] has evaluated the different traffic patterns under AODV and DSR routing protocols under RPGM Mobility Model. He concluded that AODV outperforms DSR in high load and/or high mobility situations. S.K. Singh and R. Duvvuru [4] analyzed a performance analysis of both type proactive and reactive routing protocol for ad hoc networks under CBR and TCP traffic. Their work carries a deep analysis on three important 
routing protocols AODV, DSR and DSDV and mobility models - (i) Gauss-Markov mobility model, (ii) Manhattan Grid mobility model (iii) Random Way Point mobility model and (iv) Reference Point Group mobility model. AODV protocol shows that it can perform well in dense environment but causes occasionally packet losses. AODV and DSR are found to be better than DSDV in some scenarios but still there are many challenges. S. M. D. Himabindu Pucha and Y. C. $\mathrm{Hu}$,Elizabeth Royer [5] have described in their paper about the performance of traffic patterns on routing protocols of MANETs and concluded that CBR traffic is better in reoactive routing protocols. Biradar, S. R. et. al. [6] has presented comparison study of AODV and DSR Protocol using Group Mobility Model and CBR traffic sources. They concluded that AODV performs better in average delay and high mobility is better in case of DSR for increased number of groups.

In this paper we have explored the performance of OLSR Table driven (Proactive) and TORA On-Demand (reactive) and ZRP hybrid routing protocols for performance comparison in the scenario of Random Waypoint, Manhattan, and Group Mobility Model such as military battlefield in CBR traffic. For this scenario, we have used Reference Point Group Mobility (RPGM) Model. The intention of this work is to understand their working mechanism and look into that which routing protocol gives better performance in which situation or traffic when the different Mobility Models are used for node movement.

The rest of the paper is organized as follows. The next section discusses about the OLSR, TORA and ZRP routing protocols. In section 4, we have specified the introduction of Mobility Models. Section 4 and section 5 deals with the simulation setup and results obtained on the execution of simulation. Finally, conclusion is drawn in section 6.

\section{ROUTING ALGORITHMS IN MANET}

We have used three popular routing protocols OLSR, TORA and ZRP in our simulation. The details description of all mention routing protocols are given below

\subsection{Optimized Link-State Routing (OLSR)}

Optimized Link-State Routing (OLSR) routing protocol belongs to the concept of optimization over the classical linkstate protocol tailored for operation in mobile wireless networks [7]. The key idea behind OLSR is to reduce duplicate broadcast packets in the same region. This is achieved with use of the so-called multipoint relay (MPR) nodes. Each node i selects a minimal set of multipoint relay nodes, denoted as MRP(i), from among its one-hop neighbors. The nodes in MRP(i) have the following property- every node in the symmetric two-hop neighborhood of $i$ must have a symmetric link toward MRP(i). In other words, the union of the one-hop neighbor set MRP(i) contains the whole 2-hop neighbor set. The multipoint relay selector set of a node comprises the set of neighbors that have selected it as MRP. Each node periodically floods its MRP selector set with a special type of control message called a topology control (TC) message. Using TC messages, a node announces its reachability relation to the nodes of its MRP selector set. To increase the reaction to topology changes when a change in the MRP selector is detected, the time interval between two consecutive TC message transmissions shall be decreased to a minimum.

\subsection{Temporally-Ordered Routing Algorithm (TORA)}

TORA is designed on the concept of link reversal algorithms [8][9]. The main design concept of TORA is the localization of control messages to a small set of nodes near the occurrence of a topological change. To accomplish this, nodes need to maintain routing information about their adjacent (on-hop) nodes. During the route creation and maintenance phases, nodes use a "height" metric to establish a directed acyclic graph (DAG) rooted at the destination. The DAG has the following property: there is only one sink node (the destination), while all other nodes have at least one outgoing link. TORA provides support for multiple routes. Route reconstruction is not necessary until all known routes to a destination are considered invalid. Fewer route rebuilding exercises in turn result in significant network bandwidth savings.

\subsection{Zone Routing Protocol (ZRP)}

ZRP [10][11] is a hybrid routing method, where the proactive and reactive behavior is mixed in the amounts that best match operations for an ad hoc mobile networks. Purely proactive and purely reactive protocols perform well in a limited region of this range. For example, reactive routing protocols are well suited for networks where the call-to mobility ratio is relatively low. Proactive routing protocols, on the other hand, are well suited for networks where this ratio is relatively high. There are four elements available in ZRP: MAC level function, IARP, IERP and BRP. IARP, proactive protocol is used to discover route within zone and in this case, links are considered as unidirectional. But in order to communicate with the nodes which locate in different zones, nodes use IERP, on-demand routing protocol. ZRP also follows different strategies, such as routing zone topology and proactive maintenance, for improving the efficiency and quality to discover a globally reactive route using query/reply mechanism [12].

\section{MOBILITY MODEL}

In this section, we discuss the mobility models used in our simulation study. The mobility models considered are 1.Random Waypoint Mobility Model 2.Reference Point Group Mobility Model 3.Manhattan Grid Mobility Model 


\subsection{RANDOM WAYPOINT MOBILITY MODEL}

The Random Waypoint mobility model [13] consists of pause times between changes in route and/or speed. A Mobile Node $(\mathrm{MN})$ initiated by staying in one location for a certain period of time (i.e., a pause time). Once this time finished, the Mobile Node moves to a new direction in the simulation area and a speed that is uniformly distributed between [min-speed, maxspeed]. Upon arrival, the MN pauses for a specified time period prior to starting the process again. In simulation area mobile nodes are initially distributed randomly for the most performance investigations that use the random waypoint mobility model. This initial random distribution of MNs is not delegate of the manner in which nodes distribute themselves when moving. The movement pattern of a mobile node may be influenced by and correlated with nodes in its neighborhood. In Random Waypoint each mobile node moves independently of others. Due to physical constraints of the mobile entity itself, the velocity of mobile node will change continuously and gently instead of abruptly, i.e. the current velocity is dependent on the previous velocity. However, intuitively, the velocities at two different time's slots are independent in the Random Waypoint model. In many cases the movement of a mobile node may be restricted along the street or a freeway. A geographic map may define these boundaries.

\subsection{REFERENCE POINT GROUP MOBILITY}

\section{MODEL}

The Reference Point Group Mobility (RPGM) Model [14] represents the random motion of a group of mobile nodes as well as the random motion of each individual $\mathrm{MN}$ within the group. Group movements are based upon the path travelled by a logical center or group leader for the group. The logical center determines the mobility behavior of all others nodes in the group. The movement of the group leader totally illustrates the movement of its corresponding group of mobile nodes, including their speed and direction. This model realizes the spatial dependency of each node of a group with logical center [15]. Every mobile node randomly travels about their individual pre-defined reference points, whose movements depend on the group movement. At time t the movement of group leader can be represented by Vtgroup. By some degree each member of this group deviates from this general motion Vtgroup. Based on certain pre-defined path the motion vector can be designed. The group leader is significantly affected by the movement of group members. As the individual reference points move from time $t$ to $t+1$, their locations are changed according to the group leader. The RPGM model was designed to depict scenarios such as soldiers moving together in a group in the battle field landslide rescue and During an avalanche rescue.

\subsection{MANHATTAN GRID MOBILITY MODEL}

In the Manhattan Grid Mobility Model [16], the MN movement patterns should follow a street map that represents a section of a city where the mobile ad hoc network can be applied. Here streets and speed limits on the streets are considered. At a defined point on some street each mobile network begins the simulation. A mobile node then randomly chooses a destination; also find another point on the street. The movement algorithm represents a path equivalent to the shortest travel time between the two points, one is safe driving feature like speed limit and another one is minimum distance permitted between two mobile nodes exists. All MNs must follow predefined paths and behavior guidelines (e.g. traffic laws). In the real world scenario, mobile nodes do not have the ability to roam freely without regard to obstacles and traffic regulations. By including pause times at certain intersections and destinations, incorporate acceleration and deceleration, and account for higher/lower concentrations of mobile nodes depending on the time of day we can improve the Manhattan city section mobility model.

\section{SIMULATION RESULTS AND ANALYSIS}

In this paper our target in the experiments is to study the behavior of mobility models and routing protocols under the CBR traffic model. We use Bonn Motion [17] for generating different mobility scenarios. We generate three mobility patterns with 20,50 and 100 nodes moving in an area of $1000 \mathrm{mX} 1000 \mathrm{~m}$ for a period of $1000 \mathrm{~s}$ with the first $3600 \mathrm{~s}$ of each mobility pattern ignored. The traffic generator tools cbrgen.tcl, which is a part of network simulator (ns-2) distribution, are used to generate CBR connections for $900 \mathrm{~s}$ with 1 packet/sec per source. The source and destination are chosen randomly in each traffic generator. We have used ns-2 for network simulation and traces are generated in new trace format. To compare the performance of different routing protocols under various mobility models, we have used Normalized Routing Load, Packet Delivery Fraction and average end to end delay as a performance metric.

Table -1: Simulation Parameters

\begin{tabular}{|l|l|}
\hline \multicolumn{1}{|c|}{ Parameters } & \multicolumn{1}{|c|}{ Value } \\
\hline Routing protocols & $\begin{array}{l}\text { OLSR, TORA and } \\
\text { ZRP }\end{array}$ \\
\hline Mac layer & \multicolumn{1}{|c|}{802.11} \\
\hline Packet Size & \multicolumn{1}{|c|}{512 bytes } \\
\hline Terrain Size & \multicolumn{1}{|c|}{$1000 \mathrm{mx} 1000 \mathrm{~m}$} \\
\hline Number of nodes & \multicolumn{1}{|c|}{50,150} \\
\hline Mobility Models & $\begin{array}{l}\text { Random Waypoint } \\
\text {,Manhattan Grid, } \\
\text { RPGM }\end{array}$ \\
\hline Data traffic & CBR \\
\hline Simulation time & 900 sec. \\
\hline
\end{tabular}




\begin{tabular}{|l|l|}
\hline Maximum Speed & $20 \mathrm{~m} / \mathrm{s}$ \\
\hline Minimum Speed & $5 \mathrm{~m} / \mathrm{s}$ \\
\hline Transmission Range & $500 \mathrm{~m}$ \\
\hline
\end{tabular}

We have used three mobility models and three routing protocols with varying number of sources for each type protocols. The source destination pair may be in same group or in different group. The goal of our simulation is to evaluate the performance differences of these two on-demand routing protocols. The CBR data traffic maximum numbers of sources are generated by inbuilt tool of NS-2. The parameters used for carrying out. Simulations are summarized in the table 1.

\subsection{PERFORMANCE METRICS}

Performance metrics are used to decide which routing protocol is best suitable for a mobility model. We have used following three performance metrics

\subsubsection{Normalized Routing Load:}

Normalized Routing Load (or Normalized Routing Overhead) is defined as the total number of routing packet transmitted per data packet. It is calculated by dividing the total number of routing packets sent (includes forwarded routing packets as well) by the total number of data packets received.

$$
N R L=\frac{\sum_{1}^{k} \text { Routing_Packets }}{\sum_{1}^{n} c b r_{\text {Re ceived }}}
$$

Where $c p$ sent and $c p$ forw are the control packets sent and forwarded by the router respectively and data pkt rec is the data packet received by the application.

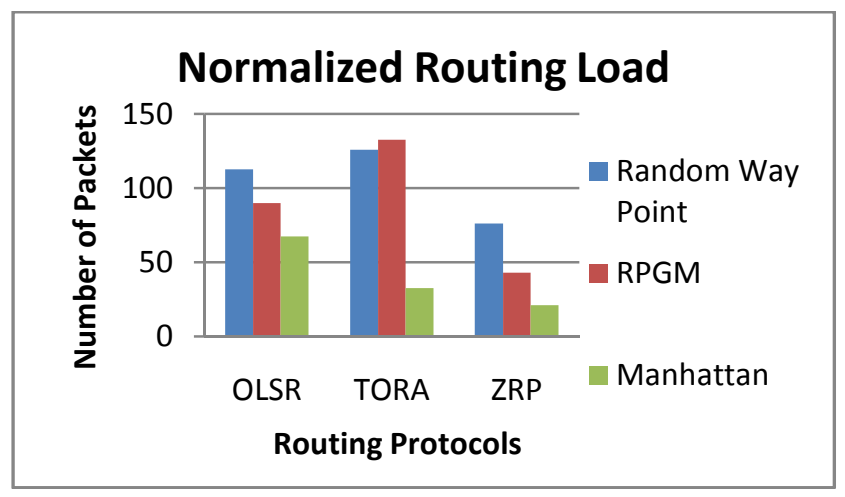

Fig. 1: Normalized Routing Load

\subsubsection{Packet Delivery Fraction}

The ratio of the data packets delivered to the destinations to those generated by the traffic generator is known as packet delivery fraction. It can be calculated as,
PDF \% $=\frac{\sum_{i}^{m} c b r_{r e c v}}{\sum_{1}^{n} c b r_{\text {sent }}} \times 100$

It can be observed from figure 1 that OLSR has best PDF traffic in all three mobility model. TORA has shown much better performance with RPGM and Random way point model. However ZRP suffers in all mobility models due to hybrid nature. Random Waypoint, which is widely used in simulation studies, does not provide a very good PDF but the variation in PDF with node density is very low in comparison of RPGM and Manhattan Grid Mobility Models.

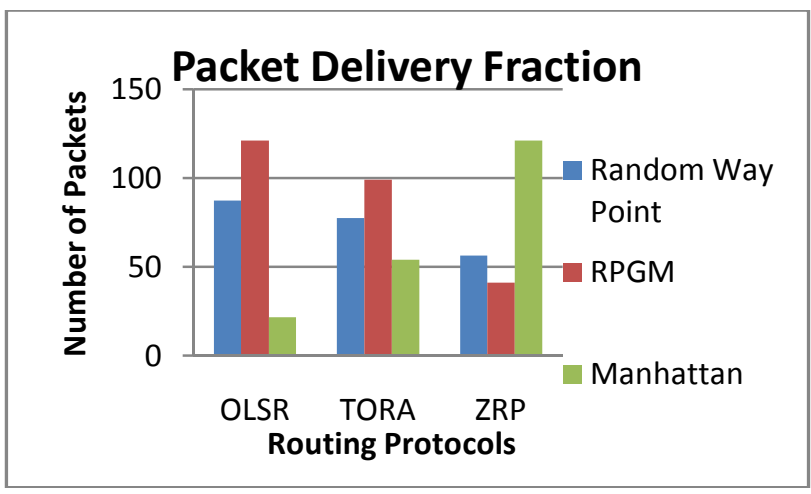

Fig. 2: Packet Delivery Fraction

In Figure 2, we can see that the simulation results of 50 nodes hybrid protocol- ZRP, reactive protocols-TORA and proactive protocol OLSR show the different characteristic in different mobility models of total received packet graph/packet delivery ratio. The ZRP graph shows that the packet delivery ratio does not fluctuate much with the increment of pause time in random way point and RPGM mobility models. ZRP delivers almost 40 percent of all packets initiated by the source at any pause time. And the variation of pause time makes a changed in averaged packet received in TORA. OLSR performance well in Random way point and RPGM mobility models. The experimental results show that for 90,120 and 150 seconds of pause times, the amount of average received packets are 337, 168 and 168 bytes/seconds respectively.

\subsubsection{A verage End-to-End Delay}

Average end to end delay includes all possible delays caused by buffering during route discovery latency, queuing at the interface queue, retransmission delays at the $\mathrm{MAC}$, and propagation and transfer times of data packets.

It is calculated for each packet id.

$\mathrm{D}=(\mathrm{Tr}-\mathrm{Ts})$

Where $\mathrm{Tr}$ is receive Time and $\mathrm{Ts}$ is sent Time 


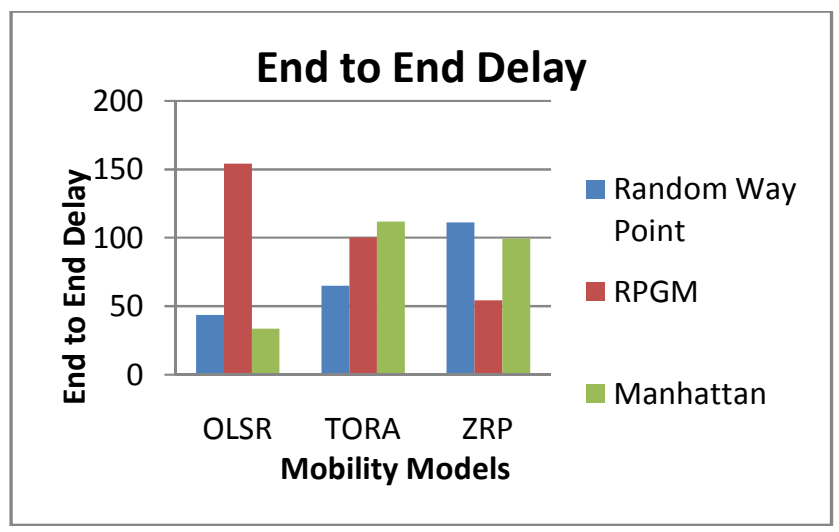

Fig. 3: End to End Delay

In Figure 3, the end-to-end graph shows that with the increment of pause time, the average end-to-end delay increases in case of ZRP but it is less at lower pause time of TORA. This graph follows the increasing trend with the increment of pause time. It is observed from above graph that RPGM outperforms in all routing protocols. Clearly the Random Waypoint exhibits the lowest delay. Among other models there is no clear winner. At lower node densities RPGM has highest delay; at medium node densities Random Waypoint is poorest while at higher node densities RPGM, Manhattan Grid provides the better results.

\section{CONCLUSIONS}

Our literature study has revealed that designing an efficient routing protocol is a fundamental issue that is very pivotal to improving the overall performance of MANET for this different mobility models play an important role. We found through our simulations results shown that the packet delivery fraction remain same across all routing protocols in random waypoint and RPGM mobility models. In case of Packet Delivery fraction, ZRP is better suited. However, TORA performs well in terms of End to End delay and Routing Overhead using either traffic types. The variation of number of nodes as well as size of area does not have much influence much on the packet delivery ratio after a certain limit. After this limit in both cases a steady level is maintained in both TORA and ZRP. It is found that Random waypoint mobility model, which is widely used in simulations of MANETs, is not a good candidate for all routing protocols. The RPGM mobility model clearly outperformed the other mobility models under the simulated scenarios. Mobility patterns are very important in evaluating the performance of ad hoc networks.

From the above discussion, we can conclude that different factors including pause time, node density and scalability have substantial influence on the overall efficiency of OLSR, TORA and ZRP routing protocols. The variations in the behavior of the different routing protocols are attributable to their different, reactive, hybrid and proactive natures. No single protocol is found to perform up to the optimum efficiency with respect to network load, throughput and packet delivery ratio for the variation of pause time, node density and network size in such dynamic, adaptive and highly variable environments.

\section{REFERENCES}

[1]. Nadia Qasim, fatin Said, Hamid Aghvami. (2009) Mobile Ad hoc Networking Protocols Evaluation through Simulation for Quality of service. IAENG International Journal of Computer Science, 36:1,IJCS_36_1_10.

[2]. F. Bai, N. Sadagopan and A. Helmy, "Important: A Framework to Systematically Analyze the Impact of Mobility on Performance of Routing Protocols for Ad Hoc Networks," IEEE Information Communications Conference (INFOCOM 2003), San Francisco, 30 March-3 April 2003, pp. 85-91.

[3]. Arindrajit Pal, Jyoti Prakash Singh, Paramartha Dutta, Paulomi Basu and Doyel Basu, "A Study on The Effect of Traffic Patterns on Routing protocols in Ad-hoc Network Following RPGM Mobility Model" Proceedings of 2011 International Conference on Signal Processing, Communication, Computing and Networking Technologies (ICSCCN 2011)

[4]. Sunil Kumar Singh, Rajesh Duvvuru and Jyoti Prakash Singh, "Performance impact of TCP and UDP on the Mobility Models and Routing Protocols in MANET". In the proc. of International Conference on Advanced Computing, Networking, and Informatics, SPRINGER, Central Institute of Technology, Raipur, Chhattisgarh, India,12-14 June, 2013 [5]. S. M. D. Himabindu Pucha and Y. C. Hu, "The performance impact of traffic patterns on routing protocols in mobile ad hoc networks," Computer Networks, vol. 51, pp. 3595-3616, 2007.

[6]. S. R. Biradar, Hiren H D Sharma, Kalpana Shrama and Subir Kumar Sarkar, "Performance Comparison of Reactive Routing Protocols of MANETs using Group Mobility Model", IEEE International Conference on Signal Processing Systems, pages 192-195 2009.

[7]. A. Qayyum, L. Viennot and A. Laouiti, "Multipoint Relaying: An Efficient Technique for Flooding in Mobile Wireless Networks," 35th Annual Hawaii International Conference on System Sciences (HICSS) 2001.

[8]. M. S. Corson, and A. Ephremides, "A Distributed Routing Algorithm for Mobile Wireless Networks," ACM / Baltzer Wireless Networks Journal, vol. 1, No. 1, pp. 61-81, 1995 [9]. V. D. Park and M. S. Corson, "A Highly Adaptive Distributed Routing Algorithm for Mobile Wireless Networks," Proceedings of the IEEE INFOCOM'97, Japan, pp. 1405-1413, 7-11 April 1997.

[10]. R. Ramanathan and M. Steenstrup. "Hierarchicallyorganized, multihop mobile wireless networks for quality-ofservice support," Mobile Networks and Applications, Vol. 3, 1998. 
[11]. E. Royer and C. K. Toh, "A Review of Current Routing Protocols for Mobile Ad-hoc Networks," IEEE Personal Communication, April 1999.

[12]. http://tools.ietf.org/id/draft-ietf-manet-zone-zrp-04.txt.

[13]. Jaya kumar. G and G. Gopinath (2008). Performance Comparison of Two On-demand Routing Protocols for Ad-hoc Networks based on Random Way Point Mobility Model". American Journal of Applied Sciences 5 (6): 2008 ISSN 15469239 (C) 2008 Science Publications. pp. 659-664.

[14]. X. Hong, M. Gerla, G. Pei and C. Chiang, "A Group Mobility Model for Ad Hoc Wireless Networks," ACM International Workshop on Modeling and Simulation of Y. ZHANG ET AL. Copyright (C) 2011 SciRes. WSN 105 Wireless and Mobile Systems (MSWiM), Seattle, 20 August 1999, pp. 53-60.

[15]. S. K. M. Harminder S. Bindra and A. L. Sangal, "Performance evaluation of two reactive routing protocols of manet using group mobility model," IJCSI International Journal of Computer Science, vol. 7, May 2010.

[16]. Tracy Camp, Jeff Boleng, Vanessa Davies. A Survey of Mobility Models for Ad Hoc Network Research. Page [119].April 12,2002.

[17]. U. of Bonn, "Bonnmotion - a mobility scenario generation and analysis tool," http://www.cs.unibonn.de/IV/bomonet/BonnMotion.htm.

\section{BIOGRAPHIES}

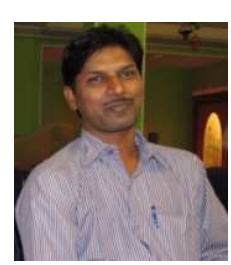

Sunil Kumar Singh is currently working as a Assistant Professor in Department of Computer Science \& Engineering at National Institute of Technology, Jamshedpur. He has a teaching experience of 4 years. He has presented nearly 6 papers in national and international conferences. His area of interest includes Computer Networks, Mobile Ad Hoc Networks and Sensor Networks.

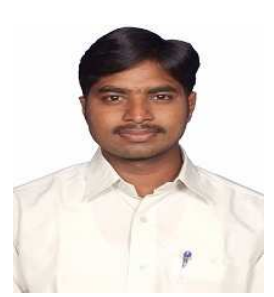

Rajesh Duvvuru is currently working as a Assistant Professor in Department of Computer Science \& Engineering at National Institute of Technology, Jamshedpur. $\mathrm{He}$ has a teaching experience of 5 years. He has published nearly 6 papers in national and international conferences and journals.

His area of interest includes Network security, cognitive radio.

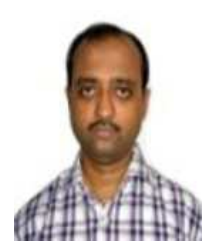

Amit Bhattcharjee is currently working as a Assistant Professor in Department of Computer Science \& Engineering at RVS College of engineering and Technology, Jamshedpur. He has a teaching experience of 2 years. His area of interest includes Mobile Ad Hoc Networks and Sensor Networks. 